\title{
Prognostic Nomogram for Overall Survival of Patients Aged 50 Years or Older with Cervical Cancer
}

\author{
Jing Yan $\mathbb{D}^{1,2}$ \\ Yue $\mathrm{He}^{\mathrm{l}}$ \\ Ming Wang' \\ Yumei $\mathrm{Wu}^{\mathrm{l}}$ \\ 'Department of Gynecological Oncology, \\ Beijing Obstetrics and Gynecology \\ Hospital, Capital Medical University; \\ Beijing Maternal and Child Health Care \\ Hospital, Beijing, People's Republic of \\ China; ${ }^{2}$ Department of Gynecology, \\ Fuxing Hospital, Capital Medical \\ University, Beijing, People's Republic of \\ China
}

Correspondence: Yumei Wu Department of Gynecological Oncology, Beijing Obstetrics and Gynecology Hospital, Capital Medical University; Beijing Maternal and Child Health Care Hospital, Dongcheng District, Qi-He-Lou Street No. 17, Beijing, People's Republic of China

Email wym597II8@ccmu.edu.cn
Objective: The prognostic factors of cervical cancer in elderly patients have not been researched systematically. We aimed to investigate the clinicopathological characteristics of patients with cervical cancer aged $\geq 50$ years and establish a nomogram for evaluating their prognoses for overall survival.

Methods: From the Surveillance, Epidemiology, and End Results database, we obtained data of 8538 patients with pathology-confirmed cervical cancer between 2004 and 2015 . Patients were divided into training $(\mathrm{n}=5941)$ and validation $(\mathrm{n}=2597)$ cohorts. A nomogram was constructed to evaluate the prognostic prediction value for disease progression. The concordance index, receiver operating characteristic curve, and calibration chart were used to evaluate the model's prediction accuracy and discriminative ability. Survival condition was analyzed using the Kaplan-Meier method.

Results: In the training cohort, age at diagnosis, race, histology, grade, stage, tumor size, number of examined lymph nodes, and treatment significantly correlated with outcome and were used to develop the nomogram. The calibration curve for survival probability showed an excellent agreement between the nomogram-predicted and actual survival in the training cohort.

Conclusion: Our nomogram has less bias and gives better accuracy than the International Federation of Gynecology and Obstetrics staging system and can help set up a more individualized feasible follow-up plan.

Keywords: prognosis, nomogram, cervical cancer, Surveillance, Epidemiology and End Results, SEER

\section{Introduction}

Cervical cancer is the most common malignant tumor of the female reproductive system, and cervical squamous cell carcinoma accounts for $90 \%$ of tumors. ${ }^{1}$ With the development of cervical cytology screening, cervical cancer and precancerous lesions can be detected and treated early. However, more than $30 \%$ of patients develop locally advanced cervical cancer in underdeveloped countries, associated with an extraordinarily low 5-year survival rate. ${ }^{2}$ A previous study suggested that the risk of cervical cancer was lower in women aged below 50 years; however, in women above 50 years, the risk of cervical cancer increased by $2 \%$ for every 1 -year age increment. ${ }^{3}$ Women aged 50 and above are usually in the perimenopausal or postmenopausal period; therefore, their physiological and pathological characteristics differ significantly from those of women in their childbearing years. 
As the population ages, the number of elderly patients increases. Most elderly patients have unapparent symptoms; therefore, the rate of treatment is low. Moreover, elderly patients have degenerative diseases, poor tolerance to surgery, and present with serious side effects following radiotherapy or chemotherapy. The additional complications in most elderly patients are difficult to treat, thus resulting in poor prognosis and high mortality. Research on the treatment outcome, survival status, and risk factors for prognosis in elderly patients is limited. Therefore, seeking to reduce the mortality of elderly patients by predicting survival, identifying prognostic factors, and developing individualized treatment for cervical cancer is crucial.

One of the most important clinical parameters for treatment determination and assessing the prognosis of cervical cancer is tumor stage. ${ }^{4}$ However, in a population with the same stage, prognosis cannot be predicted individually. According to previous literature, age, pathological type, degree of differentiation, stage, and tumor size were prognostic factors of cervical cancer. ${ }^{5-7}$ However, those factors were neither convenient nor comprehensive for treatment determination. Therefore, independent risk factors are key to an individualized prognosis assessment for patients with cervical cancer.

A nomogram is a graphical representation of logistic or Cox regression, and the application of a nomogram in cervical cancer mainly includes the prediction of recurrence and survival, ${ }^{8,9}$ risk of pelvic lymph node metastasis,$^{10}$ and risk of parametrial invasion. ${ }^{11}$ A validated nomogram can potentially be an individualized assessment tool with better accuracy and reliability for assessing the prognosis of patients with malignant tumors. In this study, we investigated the impact of clinicopathological characteristics on the survival of patients with cervical cancer aged 50 years or above and developed a nomogram that could help create feasible individualized follow-up plans.

\section{Materials and Methods}

\section{Data Source and Eligibility Criteria}

We identified cervical cancer cases from the Surveillance, Epidemiology, and End Results (SEER) database of the National Cancer Institute (http://seer.cancer.gov/). All patients with pathologically confirmed cervical cancer diagnosed at $\geq 50$ years were included. The flow chart of the patient selection process is shown in Figure 1. Finally, data of 8538 cervical cancer patients were included in this study. Patients were divided into the training cohort $(\mathrm{n}=$ 5941) and validation cohort $(\mathrm{n}=2597)$ using the random number generator of SPSS v24.0 (IBM Corp., USA). All histological types, except squamous cell carcinoma and adenocarcinoma, were recorded as "other." The surgical methods included local excision and radical surgery. Radiotherapy or chemotherapy was classified as "other" treatment. The tumor stage according to the International Federation of Gynecology and Obstetrics (FIGO) staging system (2009) was adopted.

\section{Statistical Analysis}

Categorical variables are expressed as counts and percentages, and the chi-square test was used to compare the variables. Univariate and multivariate Cox proportional hazard analyses were performed to identify the prognostic factors for overall survival (OS). The nomogram was built using significant risk factors $(\mathrm{P}<0.05)$ from the multivariate Cox analysis of the training cohort with the rms package in R v4.0.2 (http://www.r-project.org/). The predictive performance of the nomogram was measured using the $\mathrm{C}$-index, which was used to compare the nomogrampredicted survival probability with the Kaplan-Meierpredicted survival probability. The 3- and 5-year survival rate prediction abilities of the predictive models were assessed using the time receiver operating characteristic (ROC) package in R. C-index comparisons between the nomogram and FIGO staging system were performed using the rcorrp.cens package in R. The predictive performance was validated using an independent cohort.

\section{Results}

\section{Patients' Characteristics}

The training cohort included 5941 patients with cervical cancer, whereas the validation cohort included 2597 patients. The demographic and clinicopathologic characteristics of the patients in both cohorts are listed in Table 1 .

\section{Independent Prognostic Factors for Overall Survival in the Training Cohort}

Results of the univariate and multivariate analyses are listed in Table 2. Age at diagnosis, race, histology, grade, stage, tumor size, treatment, examined lymph nodes (ELNs), positive lymph nodes (PLNs), and lymph node metastasis (LNM) were associated with OS in the univariate analysis. Meanwhile, age at diagnosis, race, histology, 


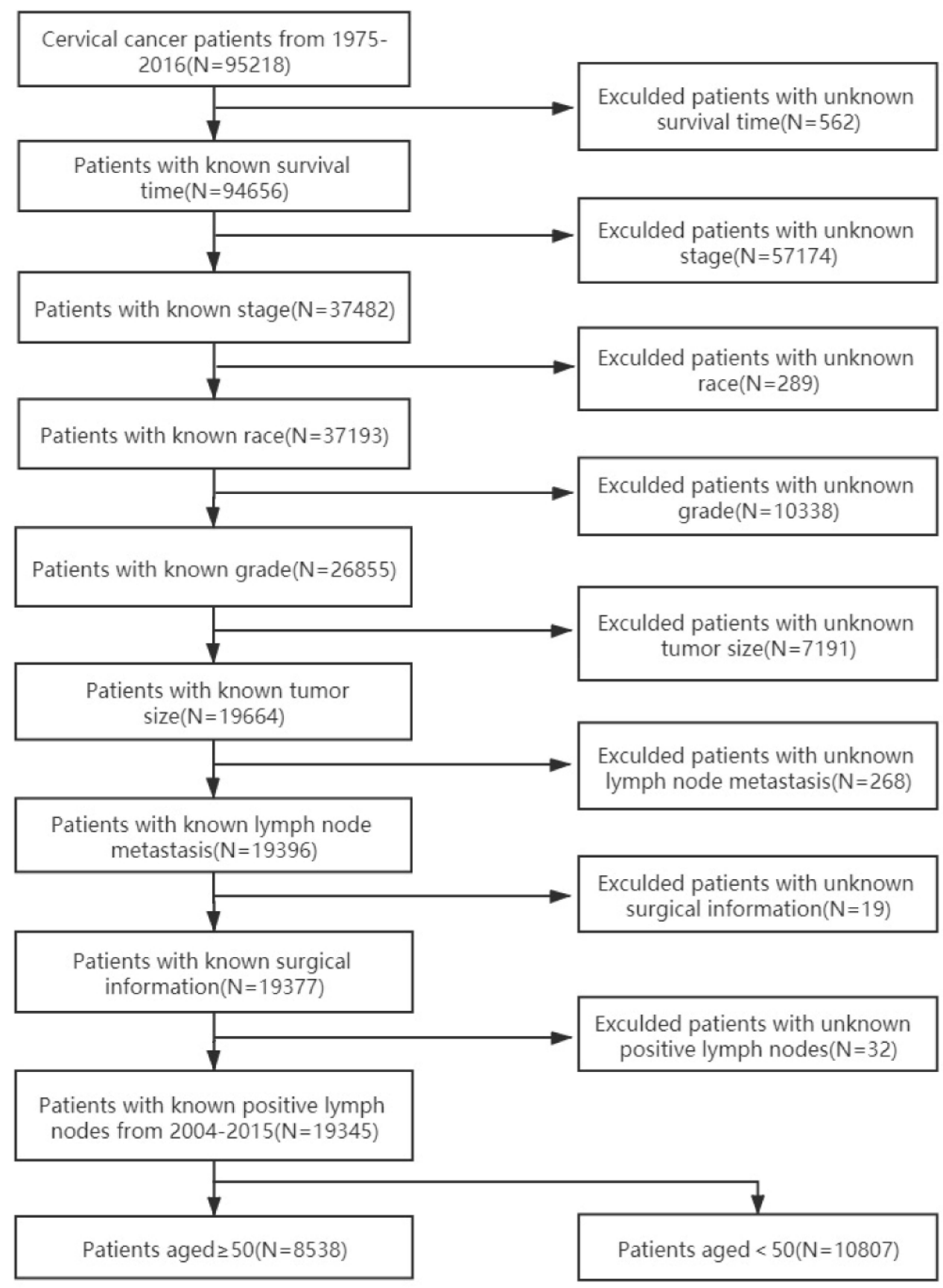

Figure I Patient selection flowchart.

grade, stage, tumor size, ELNs, and treatment were independent prognostic factors for OS in the multivariate analysis (Table 2).

\section{Prognostic Nomogram for Overall Survival}

Backward stepwise regression was used to determine the optimal model. The multivariate analysis for OS in the training cohort included significant independent prognostic factors (age at diagnosis, race, histology, grade, stage, tumor size, ELNs, and treatment) in the nomogram to predict the 3- and 5-year survival rates (Figure 2).

\section{Model Evaluation ROC Curve}

The areas under the ROC curves for predicting 3- and 5 -year survival rates were 0.818 and 0.802 , respectively 
Table I Demographic and Clinicopathological Characteristics of Patients with Cervical Cancer Between 2004 and 2015 in the SEER Database

\begin{tabular}{|c|c|c|c|c|}
\hline \multirow[t]{2}{*}{ Demographic or Characteristics } & \multirow{2}{*}{$\begin{array}{l}\text { All Subjects } \\
(\mathrm{N}=8538)\end{array}$} & \multirow{2}{*}{$\begin{array}{l}\text { Training Cohort } \\
(N=594 I)\end{array}$} & \multirow{2}{*}{$\begin{array}{l}\text { Validation Cohort } \\
(\mathrm{N}=2597)\end{array}$} & \multirow[t]{2}{*}{$P$ value } \\
\hline & & & & \\
\hline Age at diagnosis (year) & & & & 0.263 \\
\hline $50 \leq x<60$ & $4072(47.69)$ & $2808(47.26)$ & $1264(48.67)$ & \\
\hline $60 \leq x<70$ & $2576(30.17)$ & $1824(30.70)$ & $752(28.96)$ & \\
\hline$x \geq 70$ & $1890(22.14)$ & $1309(22.04)$ & $58 \mathrm{I}(22.37)$ & \\
\hline Race & & & & 0.786 \\
\hline White & $6400(74.95)$ & $4466(75.17)$ & $1934(74.47)$ & \\
\hline Black & $1122(13.14)$ & $775(13.05)$ & $347(13.36)$ & \\
\hline Other & $1016(11.89)$ & $700(11.78)$ & $316(12.17)$ & \\
\hline Histology & & & & 0.452 \\
\hline Squamous cell carcinoma & $5825(68.22)$ & $4032(67.87)$ & $1793(69.04)$ & \\
\hline Adenocarcinoma & $1892(22.16)$ & $1324(22.28)$ & $568(21.88)$ & \\
\hline Other & $821(9.62)$ & $585(9.85)$ & $236(9.08)$ & \\
\hline Grade & & & & 0.658 \\
\hline Well differentiated & $928(10.87)$ & $634(10.67)$ & $294(11.32)$ & \\
\hline Moderately differentiated & $3566(41.77)$ & $2487(4 \mid .86)$ & $1079(41.55)$ & \\
\hline Poorly differentiated & $3724(43.62)$ & $2604(43.83)$ & $1120(43.13)$ & \\
\hline Undifferentiated & $320(3.74)$ & $216(3.64)$ & $104(4.00)$ & \\
\hline Stage & & & & 0.091 \\
\hline 1 & $3512(41.13)$ & $2457(41.86)$ & $1055(40.62)$ & \\
\hline II & $1547(18.12)$ & $1087(18.09)$ & $460(\mid 7.7 I)$ & \\
\hline III & $2168(25.39)$ & $1464(25.57)$ & $704(27.11)$ & \\
\hline IV & $1311(15.36)$ & $933(14.48)$ & $378(14.56)$ & \\
\hline Tumor size $(\mathrm{cm})$ & & & & 0.198 \\
\hline$x \leq 2$ & $2184(25.58)$ & $1505(25.33)$ & $679(26.15)$ & \\
\hline $2<x<4$ & $|77|(20.74)$ & $1263(21.26)$ & $508(19.56)$ & \\
\hline$x \geq 4$ & $4583(53.68)$ & $3173(53.4 I)$ & $1410(54.29)$ & \\
\hline Surgery & & & & 0.428 \\
\hline No & $3739(43.79)$ & $2585(43.5 I)$ & II54(44.44) & \\
\hline Yes & $4799(56.21)$ & $3356(56.49)$ & $1443(55.56)$ & \\
\hline Radiation & & & & 0.987 \\
\hline No & $2812(32.94)$ & $1957(32.94)$ & $855(32.92)$ & \\
\hline Yes & $5726(67.06)$ & $3984(67.06)$ & $1742(67.08)$ & \\
\hline Chemotherapy & & & & 0.246 \\
\hline No & $3822(44.76)$ & $2684(45.18)$ & $1138(43.82)$ & \\
\hline Yes & $47 \mid 6(55.24)$ & $3257(54.82)$ & $1459(56.18)$ & \\
\hline Treatment & & & & 0.435 \\
\hline Surgery & $2174(25.46)$ & $1513(25.47)$ & $66 I(25.45)$ & \\
\hline$S+R / C$ & $2625(30.74)$ & $1843(31.02)$ & $782(30.12)$ & \\
\hline $\mathrm{R}+\mathrm{C}$ & $2740(32.09)$ & $1880(31.64)$ & $860(33.11)$ & \\
\hline Other & $695(8.15)$ & $499(8.40)$ & $196(7.55)$ & \\
\hline No & $304(3.56)$ & $206(3.47)$ & $98(3.77)$ & \\
\hline
\end{tabular}

(Continued) 
Table I (Continued).

\begin{tabular}{|c|c|c|c|c|}
\hline \multirow[t]{2}{*}{ Demographic or Characteristics } & \multirow{2}{*}{$\begin{array}{l}\text { All Subjects } \\
(\mathrm{N}=8538)\end{array}$} & \multirow{2}{*}{$\begin{array}{l}\text { Training Cohort } \\
(\mathrm{N}=594 \mathrm{I})\end{array}$} & \multirow{2}{*}{$\begin{array}{l}\text { Validation Cohort } \\
(\mathrm{N}=\mathbf{2 5 9 7})\end{array}$} & \multirow[t]{2}{*}{$P$ value } \\
\hline & & & & \\
\hline ELNs & & & & 0.289 \\
\hline $1-10$ & $979(11.47)$ & $700(11.78)$ & $279(10.74)$ & \\
\hline $1 I \leq x \leq 20$ & $|36|(\mid 5.94)$ & $955(16.07)$ & $406(15.63)$ & \\
\hline$x \geq 21$ & $1210(14.17)$ & $853(14.36)$ & $357(13.75)$ & \\
\hline Not examined & $4988(58.42)$ & $3433(57.79)$ & $1555(59.88)$ & \\
\hline PLNs & & & & 0.553 \\
\hline 0 & $2732(32.00)$ & 1929(32.47) & $803(30.92)$ & \\
\hline 1 & $367(4.30)$ & $258(4.34)$ & $109(4.20)$ & \\
\hline 2 & $176(2.06)$ & $129(2.17)$ & $47(1.81)$ & \\
\hline 3 & $24(1.45)$ & $86(1.45)$ & $38(1.46)$ & \\
\hline$\geq 4$ & $223(2.6 \mathrm{I})$ & $156(2.63)$ & $67(2.58)$ & \\
\hline Not examined & $4916(57.58)$ & $3383(56.94)$ & 1533(59.03) & \\
\hline LNM & & & & 0.880 \\
\hline No & $6224(72.90)$ & $4328(72.85)$ & 1896(73.00) & \\
\hline Yes & $2314(27.10)$ & $1613(27.15)$ & $701(27.00)$ & \\
\hline 5-year Survival & & & & 0.211 \\
\hline No & $3478(40.74)$ & $2394(40.30)$ & $1084(4 \mid .74)$ & \\
\hline Yes & $5060(59.26)$ & $3547(59.70)$ & $1513(58.26)$ & \\
\hline
\end{tabular}

Abbreviations: S, surgery; R, radiation; C, chemotherapy; ELNs, examined lymph nodes; PLNs, positive lymph nodes; LNM, lymph node metastasis.

as shown in Figure 3, in the training cohort (3a and $\mathbf{3 b}$ ) and 0.838 and 0.813 , respectively, in the validation cohort (3c and 3d).

\section{Calibration Plot}

The calibration plot for the probability of survival at 3 or 5 years showed an optimal agreement between the prediction of the nomogram and the actual observation as shown in Figure 4 in training cohorts $(\mathbf{4 a}$ and $\mathbf{4 b})$ and validation cohorts (4c and $\mathbf{4 d})$.

\section{Comparison with FIGO Stage System}

The C-index values for FIGO staging and nomogram were 0.719 (95\% confidence interval [CI]: 0.709 to 0.728 ) and 0.788 (95\% CI, 0.778 to 0.797$)$ in the training cohort $(\mathrm{P}<0.01)$, respectively, and 0.732 (95\% CI: 0.718 to 0.745$)$ and 0.791 (95\% CI, 0.777 to 0.804$)$ in the validation cohort $(\mathrm{P}<0.01)$, respectively. These results show that the prediction model had a moderate accuracy and that the prediction results were more reliable than those of the traditional FIGO staging system.

\section{Survival Analysis}

Kaplan-Meier survival analysis of OS for different clinicopathological characteristics of patients with cervical cancer in the training cohort are shown in Figure 5.

\section{Discussion}

The incidence of cervical cancer is increasing, and the average age at onset has dropped from 60 years in the early 20 th century to 50 years. ${ }^{12}$ Patients aged 50 years and above have the highest mortality rate. The incidences of senile vaginitis and cervical inflammation are high among patients with unapparent symptoms. However, some patients ignore their symptoms and consult late. Thus, the elderly population has a higher proportion of patients with advanced cervical cancer. Of the 8538 patients included in this study, 3479 (40.74\%) had stage III-IV disease, and 4583 (53.67\%) had tumors larger than $4 \mathrm{~cm}$.

The incidence of cervical cancer is increasing, and the average age at onset has dropped from 60 years in the early 20th century to 50 years. ${ }^{12}$ The analysis may be partly related to widespread cervical cancer screening and regular care with a primary care physician who does cervical cancer screening. Patients aged 50 years and above have the highest mortality rate. The incidences of senile vaginitis and cervical inflammation are high among patients with unapparent symptoms. However, some patients ignore their symptoms and consult late. Thus, the elderly population has a higher proportion of patients 
Table 2 Univariate Analysis and Multivariate Analysis in the Training Cohort

\begin{tabular}{|c|c|c|c|c|}
\hline \multirow[t]{2}{*}{ Variables } & \multicolumn{2}{|c|}{ Univariate Analysis } & \multicolumn{2}{|c|}{ Multivariate Analysis } \\
\hline & HR (95\% Cl) & $P$ value & HR (95\% Cl) & $P$ value \\
\hline \multicolumn{5}{|l|}{ Age at diagnosis (year) } \\
\hline $50 \leq x<60$ & Reference & & Reference & \\
\hline $60 \leq x<70$ & $1.229(1.114-1.356)$ & $<0.001$ & $1.229(1.112-1.357)$ & $<0.001$ \\
\hline$X \geq 70$ & $2.30 I(2.09 \mid-2.532)$ & $<0.001$ & $1.625(1.210-2.182)$ & $<0.001$ \\
\hline \multicolumn{5}{|l|}{ Race } \\
\hline White & Reference & & Reference & \\
\hline Black & $1.419(1.273-1.583)$ & $<0.001$ & $1.206(1.080-1.347)$ & $<0.001$ \\
\hline Other & $0.806(0.702-0.925)$ & 0.002 & $0.815(0.709-0.937)$ & 0.004 \\
\hline \multicolumn{5}{|l|}{ Histology } \\
\hline Squamous cell carcinoma & Reference & & Reference & \\
\hline Adenocarcinoma & $0.825(0.744-0.914)$ & $<0.001$ & $1.235(1.109-1.376)$ & $<0.001$ \\
\hline Other & $1.392(1.229-1.577)$ & $<0.001$ & $1.321(1.160-1.505)$ & $<0.001$ \\
\hline \multicolumn{5}{|l|}{ Grade } \\
\hline Well differentiated & Reference & & Reference & \\
\hline Moderately differentiated & $1.629(1.370-1.937)$ & $<0.001$ & $1.472(\mid .23 \mathrm{I}-1.76 \mathrm{I})$ & $<0.001$ \\
\hline Poorly differentiated & $2.482(2.095-2.940)$ & $<0.001$ & $1.696(I .42 I-2.024)$ & $<0.001$ \\
\hline Undifferentiated & $3.173(2.497-4.032)$ & $<0.001$ & $2.013(1.573-2.576)$ & $<0.001$ \\
\hline \multicolumn{5}{|l|}{ Stage } \\
\hline 1 & Reference & & Reference & \\
\hline II & $2.230(1.963-2.534)$ & $<0.001$ & I.484(I.284-I.7I5) & $<0.001$ \\
\hline III & $3.250(2.902-3.640)$ & $<0.001$ & $2.531(2.166-2.956)$ & $<0.001$ \\
\hline IV & $8.075(7.198-9.060)$ & $<0.001$ & $4.522(3.894-5.321)$ & $<0.001$ \\
\hline \multicolumn{5}{|l|}{ Tumor size $(\mathrm{cm})$} \\
\hline$x \leq 2$ & Reference & & Reference & \\
\hline $2<x<4$ & $1.858(1.596-2.162)$ & $<0.001$ & $1.363(1.160-1.602)$ & $<0.001$ \\
\hline$x \geq 4$ & $4.084(3.600-4.633)$ & $<0.001$ & $1.843(1.584-2.144)$ & $<0.001$ \\
\hline \multicolumn{5}{|l|}{ Treatment } \\
\hline No & Reference & & Reference & \\
\hline Surgery & $0.056(0.046-0.068)$ & $<0.001$ & $0.297(0.237-0.372)$ & $<0.001$ \\
\hline$S+R / C$ & $0.105(0.089-0.124)$ & $<0.001$ & $0.24 I(0.200-0.290)$ & $<0.001$ \\
\hline $\mathrm{R}+\mathrm{C}$ & $0.191(0.163-0.224)$ & $<0.001$ & $0.216(0.183-0.255)$ & $<0.001$ \\
\hline Other & $0.450(0.377-0.537)$ & $<0.001$ & $0.460(0.384-0.550)$ & $<0.001$ \\
\hline \multicolumn{5}{|l|}{ ELNs } \\
\hline $1-10$ & Reference & & Reference & \\
\hline $\mid I \leq x \leq 20$ & $0.68 I(0.564-0.822)$ & $<0.001$ & $0.732(0.605-0.885)$ & 0.001 \\
\hline$x \geq 21$ & $0.576(0.472-0.704)$ & $<0.001$ & $0.628(0.5 \mid 2-0.769)$ & $<0.001$ \\
\hline Not examined & $2.114(1.837-2.434)$ & $<0.001$ & $1.493(0.970-2.296)$ & 0.067 \\
\hline \multicolumn{5}{|l|}{ PLNs } \\
\hline 0 & Reference & & Reference & \\
\hline 1 & $2.011(1.592-2.540)$ & $<0.001$ & $0.792(0.609-1.031)$ & 0.083 \\
\hline 2 & $2.225(1.656-2.990)$ & $<0.001$ & $0.918(0.666-1.266)$ & 0.605 \\
\hline 3 & $2.645(1.902-3.680)$ & $<0.001$ & $0.995(0.70 I-I .4 \mid 3)$ & $0.98 \mathrm{I}$ \\
\hline$\geq 4$ & $3.68 I(2.903-4.666)$ & $<0.001$ & $1.275(0.979-1.660)$ & $0.07 I$ \\
\hline Not examined & $3.84 I(3.433-4.299)$ & $<0.001$ & $0.989(0.652-1.500)$ & 0.959 \\
\hline
\end{tabular}

(Continued) 
Table 2 (Continued).

\begin{tabular}{|l|l|l|l|l|}
\hline \multirow{2}{*}{ Variables } & \multicolumn{2}{l|}{ Univariate Analysis } & \multicolumn{2}{l|}{ Multivariate Analysis } \\
\cline { 2 - 5 } & HR (95\% Cl) & P value & HR (95\% Cl) & P value \\
\hline LNM & & & & Reference \\
No & Reference & $<0.001$ & $1.067(0.949-1.200)$ & 0.273 \\
Yes & $2.288(2.107-2.486)$ & & \\
\hline
\end{tabular}

Abbreviations: HR, hazard ratio; $\mathrm{Cl}$, confidence interval; ELNs, examined lymph nodes; PLNs, positive lymph nodes; LNM, lymph node metastasis.

with advanced cervical cancer. Of the 8538 patients included in this study, 3479 (40.74\%) had stage III-IV disease, and 4583 (53.67\%) had tumors larger than $4 \mathrm{~cm}$. As cervical cancer is the only preventable cancer, regular cervical cancer screening can detect early and reduce the mortality of cervical cancer patients. ${ }^{13}$

We analyzed the prognostic factors of cervical cancer in elderly patients. Results showed that age, race, histology, grade, stage, tumor size, ELNs, and treatment were independent prognostic factors $(\mathrm{P}<0.05)$. As patients age, the disease progresses, patients' physical state declines, and lymph nodes metastasis occurs, resulting in poor prognosis and a shortened survival period. We also observed that black patients had a much higher risk of death than the whites and other races, which could be related to the patient's financial status, educational level, environment, diet, and health care. However, this study was based on the
SEER database, which involved mainly Caucasians (74.95\% of the population). Therefore, more evidence is needed to confirm the difference in the prognosis of cervical cancer among different ethnic groups.

Tumor size, an important index in the evaluation of local primary cervical carcinoma, has a long-standing influence on cervical cancer prognosis. Chang et $\mathrm{al}^{14}$ analyzed the data of 797 patients and found that tumor diameter $>4 \mathrm{~cm}$ was an independent risk factor of recurrence and death. However, we observed that tumor size $>2 \mathrm{~cm}$ was an independent survival factor. Poor differentiation often indicates a high degree of malignancy and strong aggressiveness, but there is still no complete consensus on this. Some studies have shown that a low degree of tumor differentiation has no effect on prognosis ${ }^{15}$ while others have suggested that a combination of histological differentiation and pelvic lymph node metastasis can affect

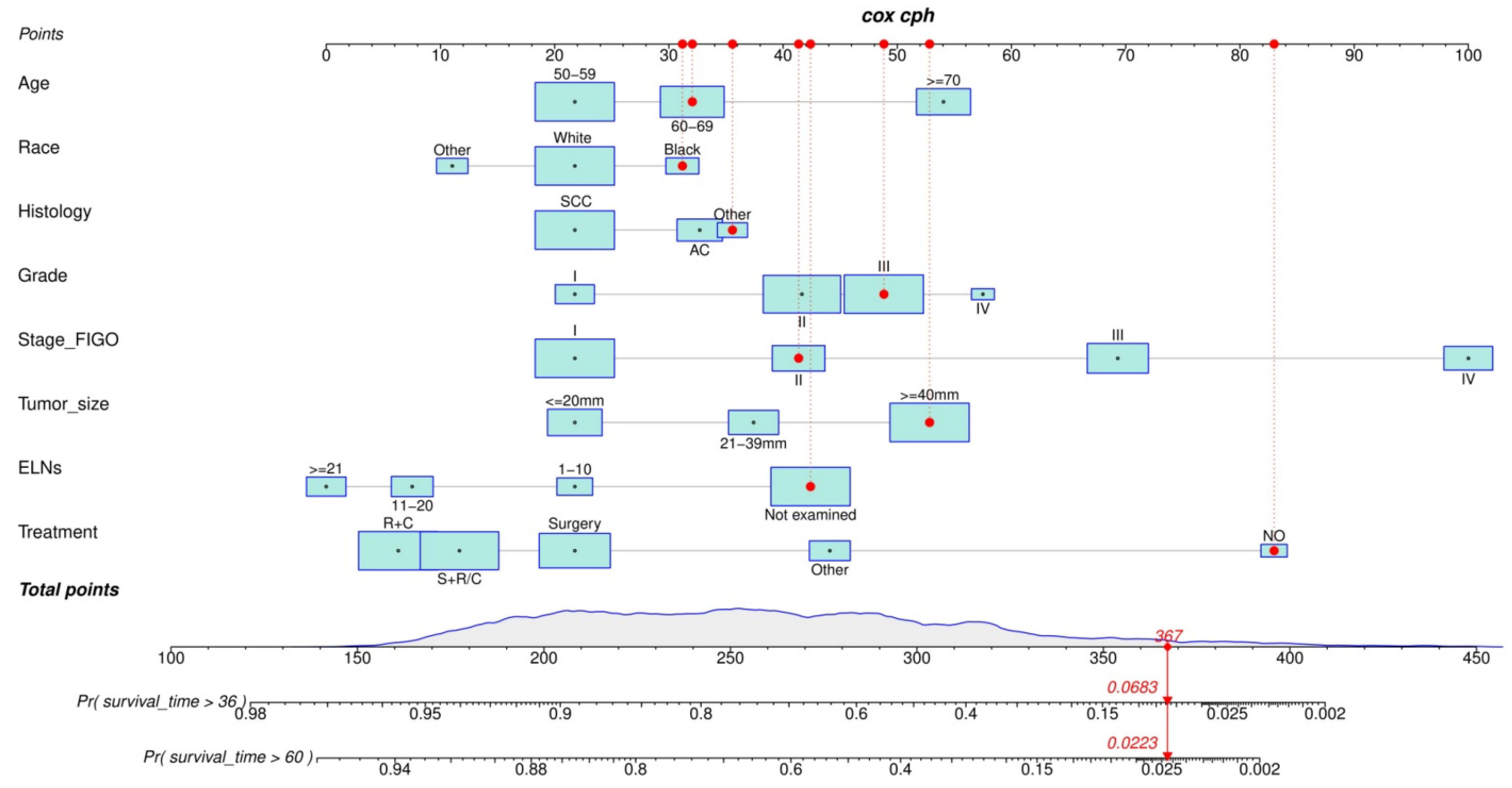

Figure 2 Cervical cancer survival nomogram.

Abbreviations: SCC, squamous cell carcinoma; AC, adenocarcinoma. 


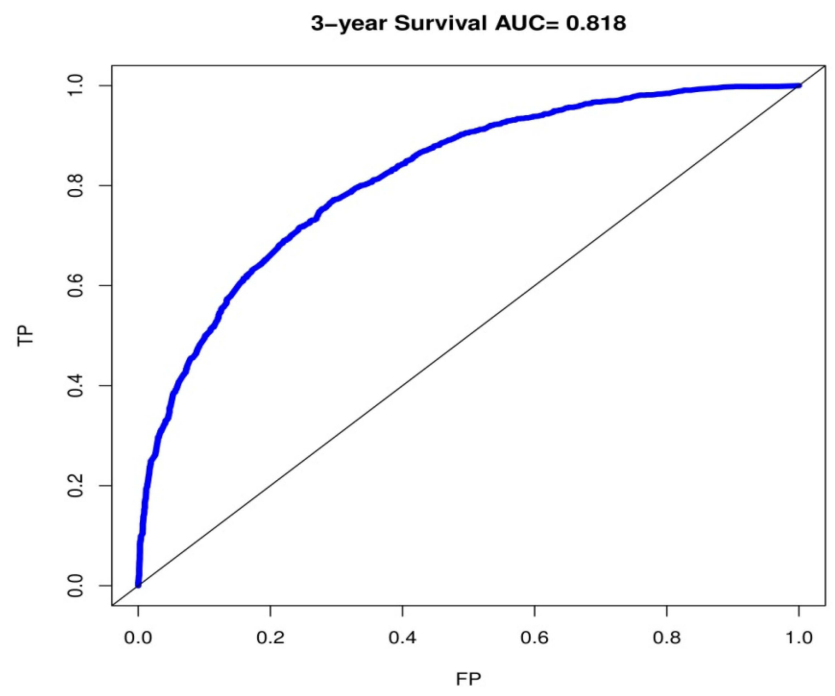

A

3-year Survival AUC $=0.838$

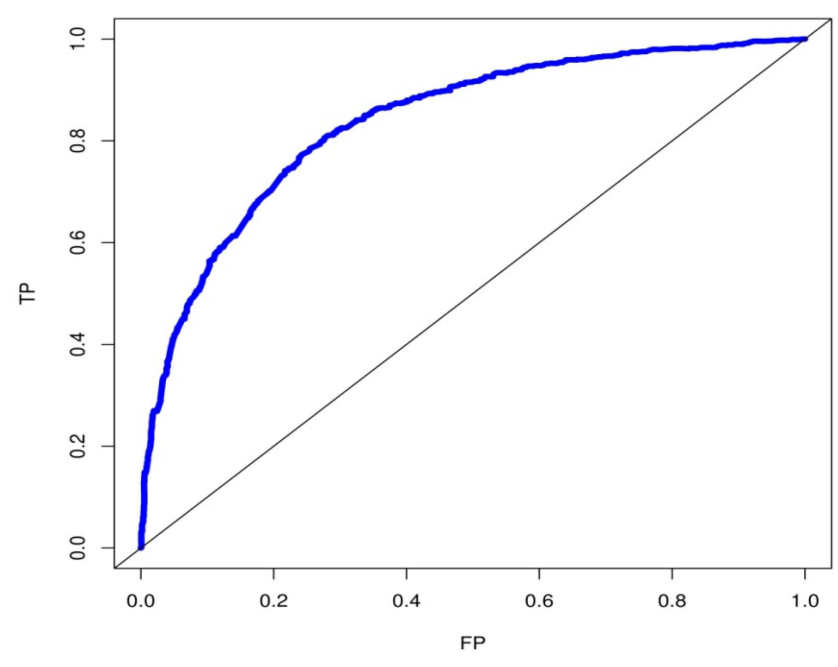

C 5-year Survival AUC $=0.80242$

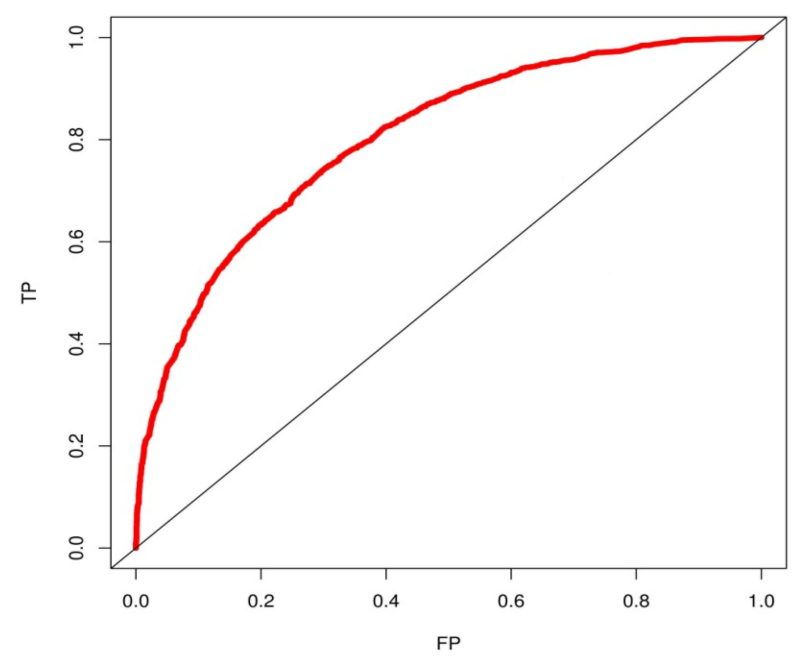

B

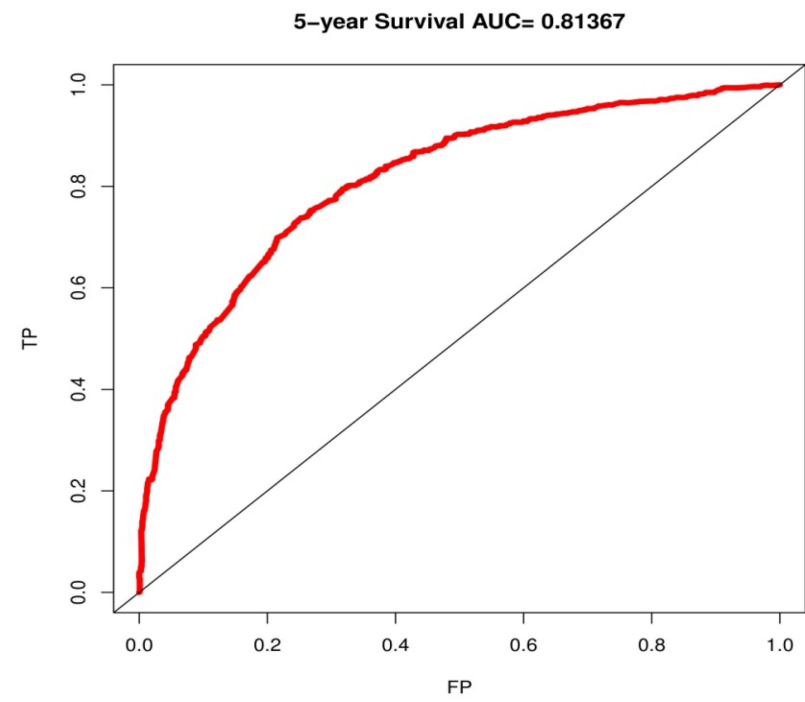

D

Figure 3 The ROC curve for predicting patient survival at (A) 3 years and (B) 5 years in the training cohort and at (C) 3 years and (D) 5 years in the validation cohort. The false positive (FP) rate is plotted on the $\mathrm{X}$-axis, and the true positive (TP) rate is plotted on the $\mathrm{Y}$-axis.

Abbreviation: AUC, area under the curve.

prognosis. ${ }^{16}$ In our study, tumor grade, histology, PLNs, and LNM affected survival in univariate analysis, but PLNs and LNM were not independent factors in multivariate analysis. The reason mainly lies in the large proportion of patients with large tumor diameter in the included cases. Since lymph node metastasis is closely related to tumor size, our study may reduce the influence of lymph node metastasis for OS, but this still needs further study and verification. In addition, as PLNs are related to the number of lymph nodes dissected during surgery ${ }^{17}$ using PLNs to evaluate the prognosis of cervical cancer is not objective. Thus, some scholars have proposed the inclusion of positive lymph node ratio as a prognostic factor of early cervical cancer. ${ }^{18}$

The National Comprehensive Cancer Network guidelines recommend surgery as the main treatment option for patients with early-stage cervical cancer and a combination of surgery and concurrent chemoradiotherapy in patients with advanced disease in the United States. ${ }^{19,20}$ The survival analysis in this study showed that treatment with surgery and ELNs $\geq 21$ were associated with a high survival rate, indicating that surgery is the optimal treatment for patients with earlystage cervical cancer and that an extensive metastatic 
lymph node resection should be performed. However, complete lymph node resection is challenging in elderly patients as they often have chronic diseases and poor physical status. Therefore, adjuvant radiotherapy and chemotherapy are indicated postoperatively in these patients. Unlike young patients, elderly patients respond poorly and show partial resistance to radiotherapy. ${ }^{21}$ This is mainly due to low estrogen levels, vaginal mucosa atrophy, and vaginal stenosis caused by cervical lesions invading the vaginal wall in elderly patients. During radiotherapy, placing the after loading device in a fixed position is difficult, resulting in an inaccurate distribution of the dose and target area. Moreover, radiotherapy is ineffective for patients with possible lymph node metastasis.

Cervical cancer is relatively insensitive to chemotherapy, and chemotherapy is only used as part of the comprehensive treatment for advanced and recurrent cases. Lymph node metastasis is the most common mechanism of cervical cancer metastasis, and once it occurs, the 5-year survival rate of patients is reduced from $85-90 \%$ to $50-55 \% .^{22}$ The lymph node metastasis rate in the elderly patients with cervical cancer in this study was $27.10 \%$, and univariate analysis suggested that pelvic lymph node metastasis was an important prognostic factor. Therefore, the role of chemotherapy

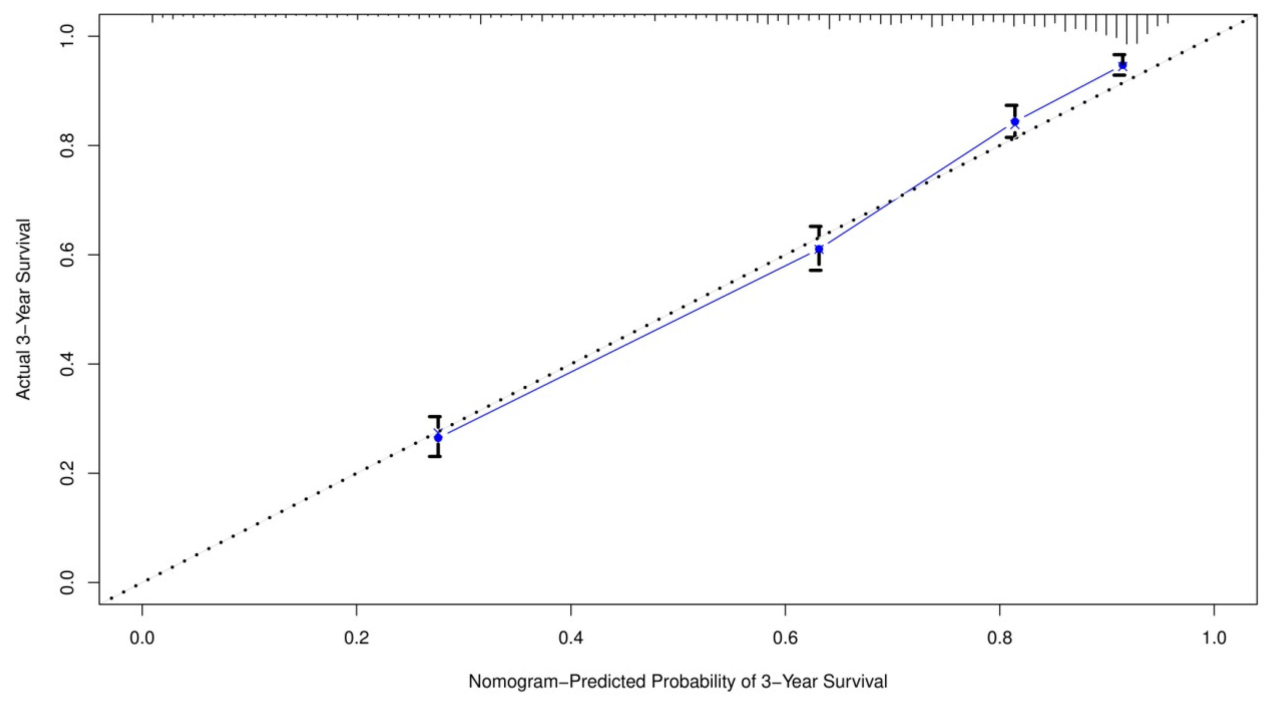

A

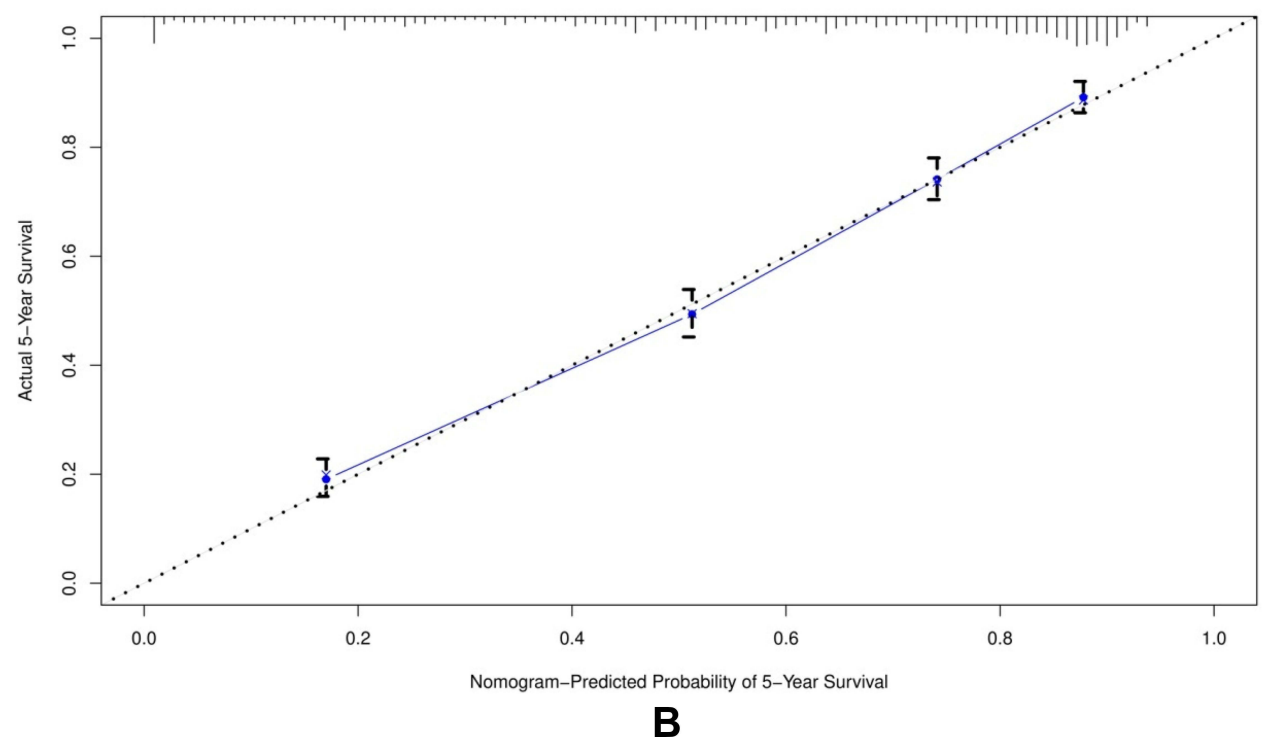

Figure 4 Continue. 

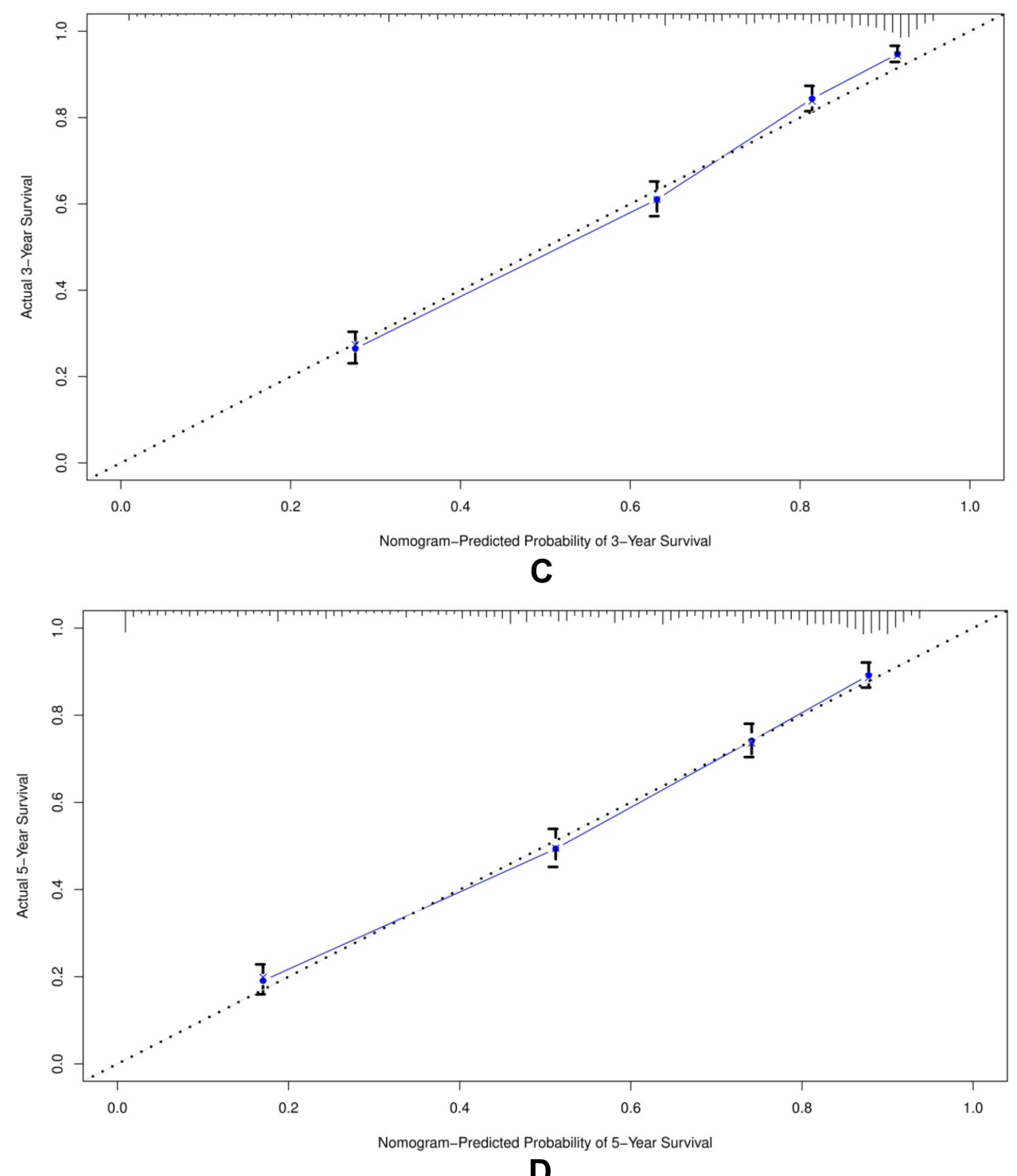

Figure 4 The calibration curve for predicting patient survival at (A) 3 years and (B) 5 years in the training cohort, and at (C) 3 years and (D) 5 years in the validation cohort. The nomogram-predicted probability of overall survival is plotted on the $\mathrm{X}$-axis, the actual overall survival is plotted on the $\mathrm{Y}$-axis.

in elderly patients with cervical cancer should not be ignored. In our study sample, patients who received chemotherapy treatment accounted for $55.24 \%$, indicating that chemotherapy was also a main therapeutic treatment for this group. When chemotherapy is used, radiotherapy-related ovarian and vaginal injury is bypassed and surgical safety is improved. Therefore, chemotherapy can be used for individualized cervical cancer treatment. However, as the adverse effects of chemotherapy drugs cannot be completely avoided, the upper age limit for chemotherapy administration as well as the standard chemotherapy dose and regimen for elderly patients is still pending.
Most women aged over 70 years receive either no treatment or non-aggressive treatment, regardless of their tumor stage. Our results showed that elderly women gained a significant survival advantage from treatment, even with less aggressive treatment $(\mathrm{p}<0.001)$. Meanwhile, multivariate analysis showed that those who received chemoradiotherapy had the greatest survival advantage (hazard ratio $=0.216, \mathrm{P}<0.001$ vs no treatment). Previous studies have shown that concurrent cisplatin-based chemoradiotherapy can improve the survival rate of patients with cervical cancer and can reduce their mortality by $30-50 \% .^{23}$ Therefore, treatment methods should vary based on the patient characteristics. 
Surival curve of Age $(p$ Value $=0)$

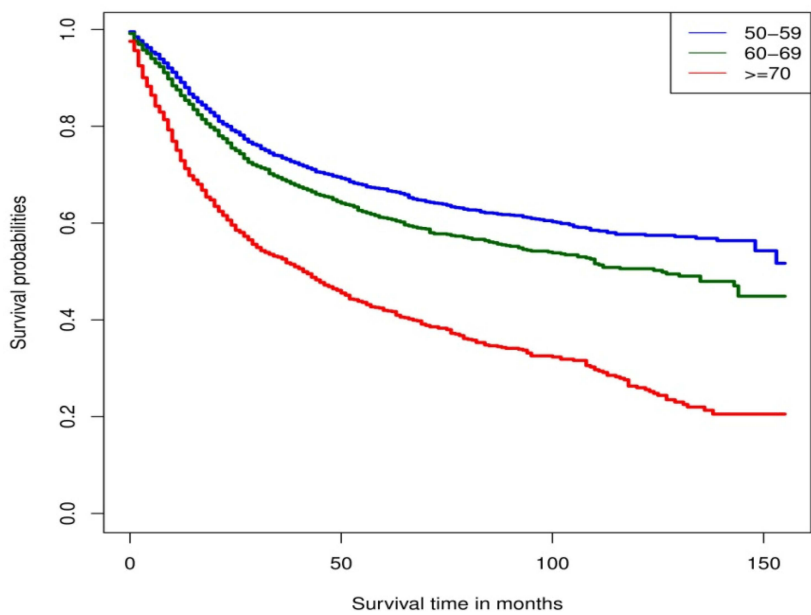

A

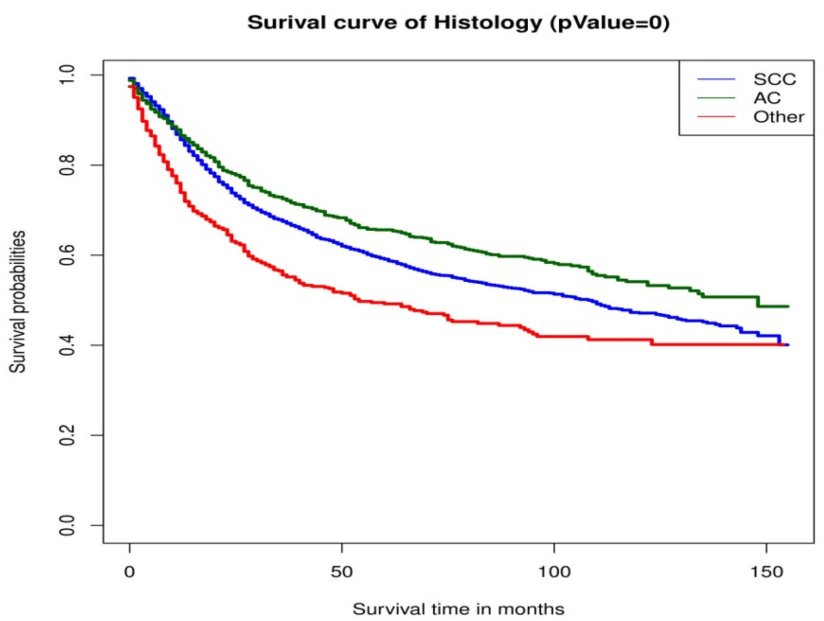

C

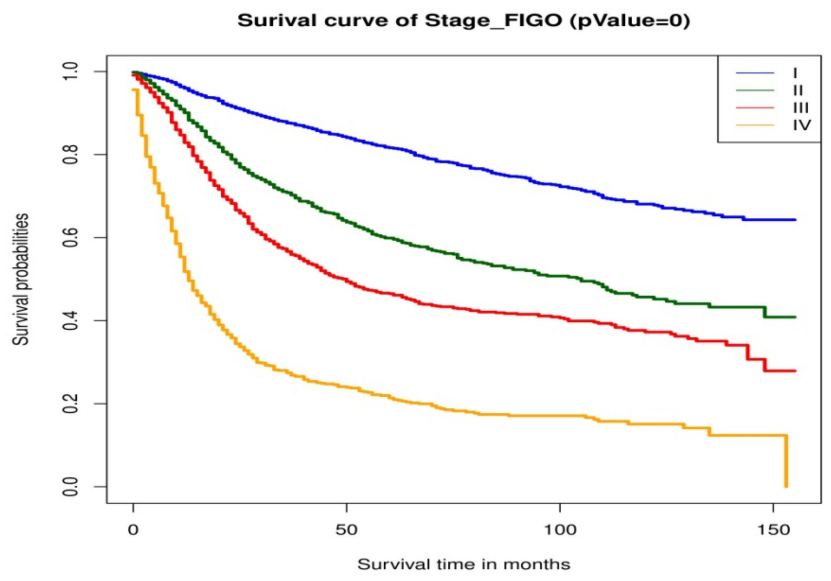

E
Surival curve of Race $(p$ Value $=0)$

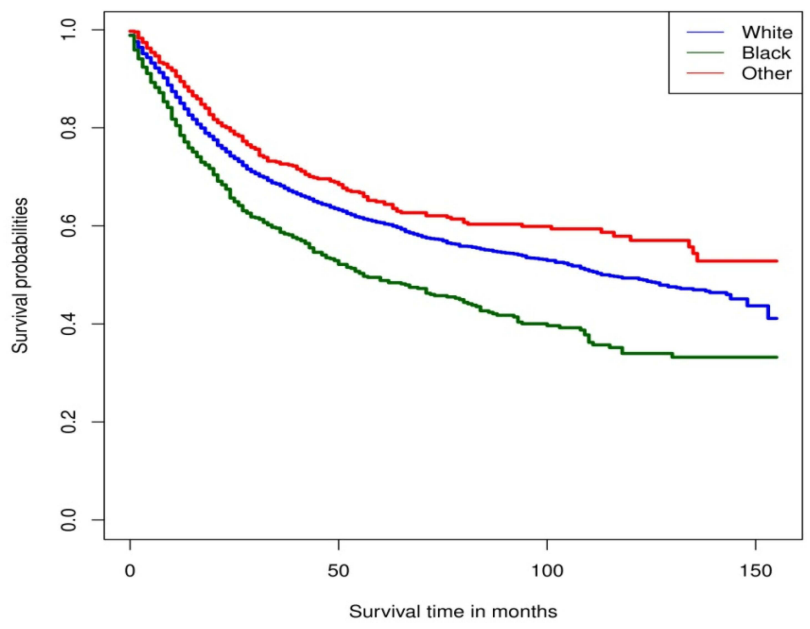

B

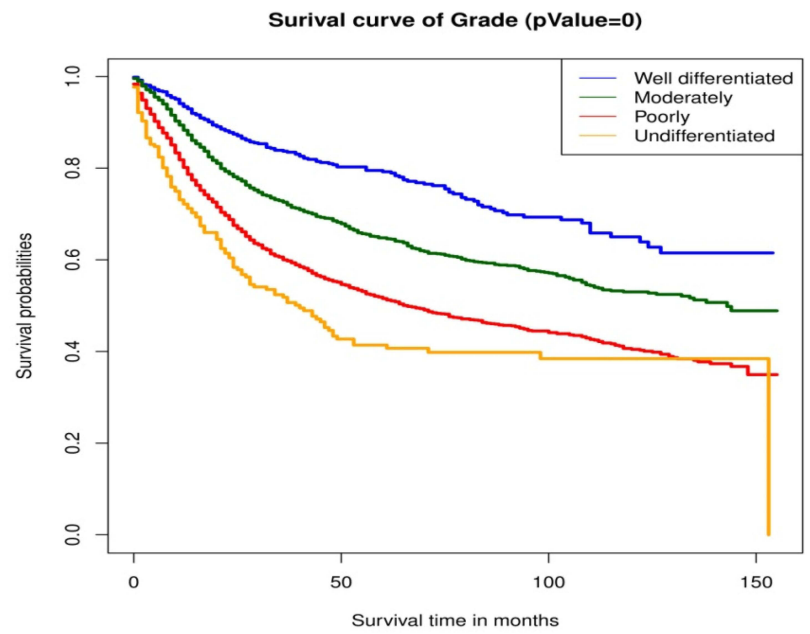

D

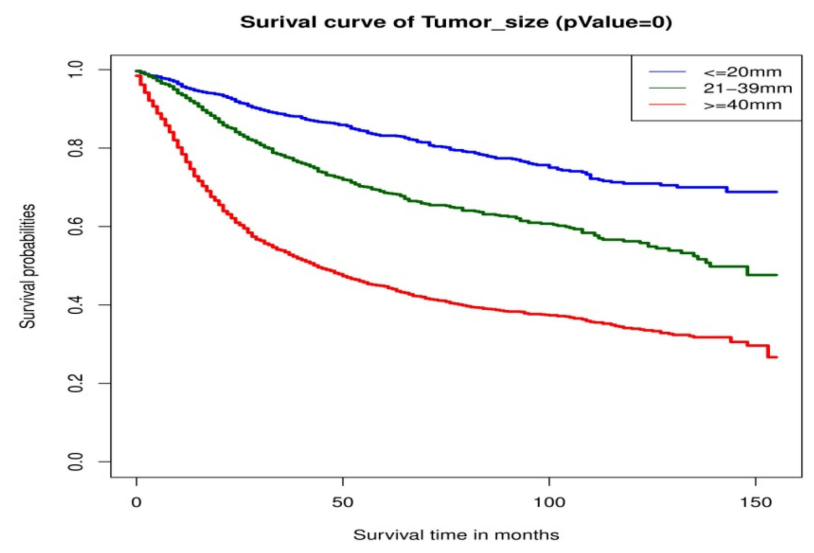

$\mathbf{F}$

Figure 5 Continue. 
Surival curve of ELNs (pValue=0)

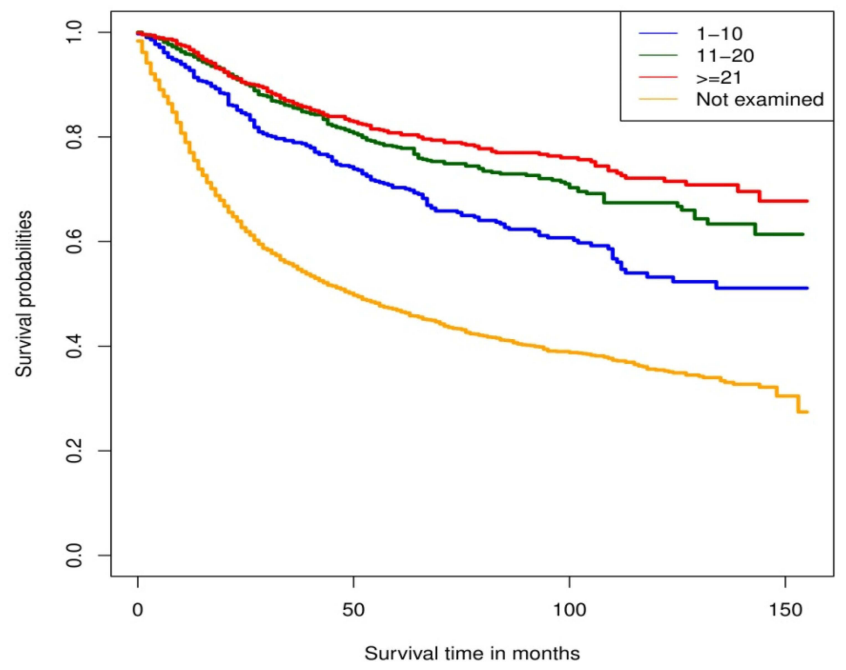

G
Surival curve of PLNs (pValue=0)

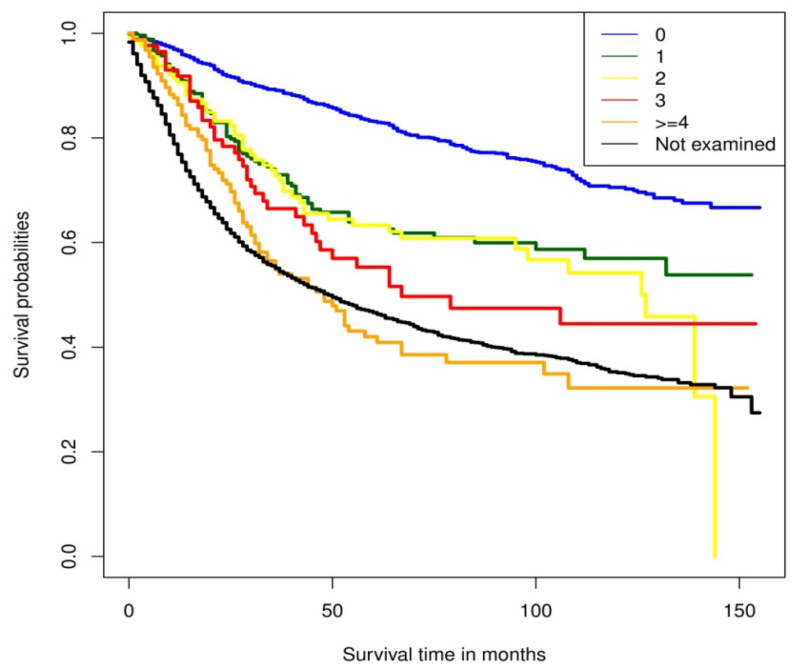

H

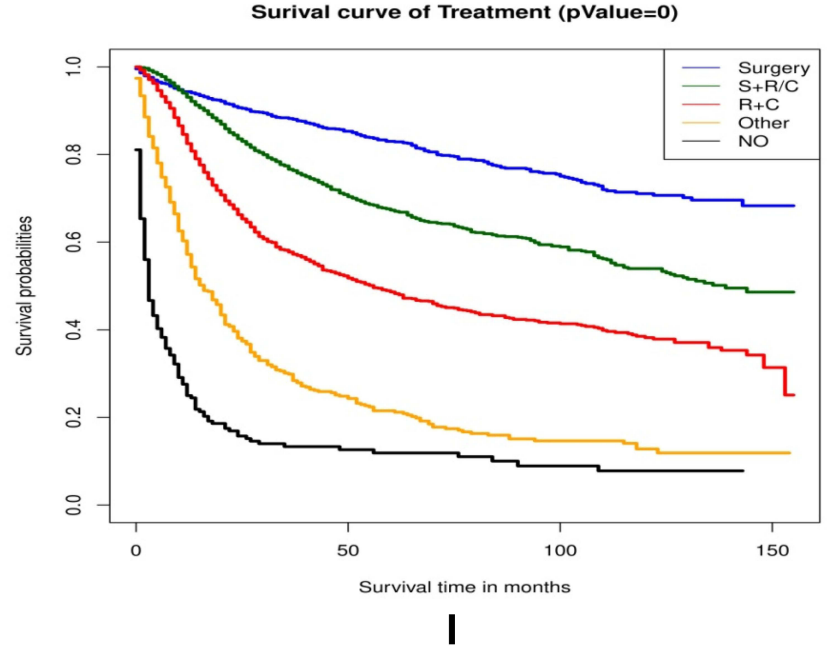

Figure 5 The Kaplan-Meier survival curves in the training cohort are shown in (A-I). The results demonstrated that patients with age under 70 (A), non-black race (B), well-differentiated grade (C), squamous cell carcinoma and adenocarcinoma (D), early FIGO stage (E), small tumor size (F), more ELNs (G), negative lymph node (H), and receiving treatment $(\mathbf{I})$ had better survival time $(\mathrm{P}=0)$.

Thus far, there is limited research on elderly patients with cervical cancer regarding their characteristics and treatments. Our study proposed a highly reliable nomogram model for elderly patients with cervical cancer, which has a better accuracy than the FIGO staging system. However, our study has some limitations. First, the majority of the women included in the SEER database are Caucasian. Moreover, our nomogram was only verified internally, but not externally. Finally, we were unable to collect information from the SEER database regarding the general condition and complications in elderly patients, which could have influenced treatment choices, made by patients and caused bias within our results.

\section{Conclusions}

This study established an easy-to-use nomogram made up of independent prognostic factors of cervical cancer in patients aged 50 years or above. The evaluation results verify the reliability and validity of the model, which is valuable in formulating treatment plans and evaluating individual prognoses.

\section{Ethics Approval}

This study was approved by the ethics committees of Fuxing Hospital, Capital Medical University having approval no CR020210801 and study was conducted in accordance with the Declaration of Helsinki. The 
information was taken from the Surveillance, Epidemiology, and End Results (SEER) database of the National Cancer Institute https://seer.cancer.gov/data/.

\section{Acknowledgments}

We are thankful to Beijing Municipal Administration of Hospitals Clinical Medicine Development of Special Funding for supporting our research. We are also thankful to all the authors for their invaluable contribution to this study.

\section{Author Contributions}

All authors made a significant contribution to the work reported, whether that is in the conception, study design, execution, acquisition of data, analysis and interpretation, or in all these areas; took part in drafting, revising or critically reviewing the article; gave final approval of the version to be published; have agreed on the journal to which the article has been submitted; and agree to be accountable for all aspects of the work.

\section{Funding}

This research was supported by Beijing Municipal Administration of Hospitals Clinical Medicine Development of Special Funding Support (ZYLX201705).

\section{Disclosure}

The authors declare no conflicts of interest, financial or otherwise.

\section{References}

1. alys-Tagoe BNL, Aheto JMK, Mensah G, Biritwum RB, Yawson AE. Cervical cancer screening practices among women in Ghana: evidence from wave 2 of the WHO study on global AGEing and adult health. $B M C$ Women's Health. 2020;20(1):49. doi:10.1186/s12905-020-00915-9.

2. de Martel C, Georges D, Bray F, Ferlay J, Clifford GM. Global burden of cancer attributable to infections in 2018: a worldwide incidence analysis. Lancet Glob Health. 2020;8(2):e180-e190. doi:10.1016/ S2214-109X(19)30488-7.

3. Moore KN, Java JJ, Slaughter KN, et al. Is age a prognostic biomarker for survival among women with locally advanced cervical cancer treated with chemoradiation? An NRG Oncology/Gynecologic Oncology Group ancillary data analysis. Gynecol Oncol. 2016;143 (2):294-301. doi:10.1016/j.ygyno.2016.08.317.

4. Polterauer S, Grimm C, Hofstetter G, et al. Nomogram prediction for overall survival of patients diagnosed with cervical cancer. $\mathrm{Br}$ J Cancer. 2012;107(6):918-924. doi:10.1038/bjc.2012.340.

5. Chen CC, Wang L, Lin JC, Jan JS. The prognostic factors for locally advanced cervical cancer patients treated by intensity-modulated radiation therapy with concurrent chemotherapy. J Formos Med Assoc. 2015;114(3):231-237. doi:10.1016/j.jfma.2012.10.021.

6. Winer I, Alvarado-Cabrero I, Hassan O, et al. The prognostic significance of histologic type in early stage cervical cancer - A multi-institutional study. Gynecol Oncol. 2015;137(3):474-478. doi:10.1016/j.ygyno.2015.02.005.
7. Cui L, Shi Y, Zhang GN. Perineural invasion as a prognostic factor for cervical cancer: a systematic review and meta-analysis. Arch Gynecol Obstet. 2015;292(1):13-19. doi:10.1007/s00404-015-3627-z.

8. Ouyang D, Yang P, Cai J, Sun S, Wang Z. Comprehensive analysis of prognostic alternative splicing signature in cervical cancer. Cancer Cell Int. 2020;20:221. doi:10.1186/s12935-020-01299-4.

9. Yang J, Tian G, Pan Z, et al. Nomograms for predicting the survival rate for cervical cancer patients who undergo radiation therapy: a SEER analysis. Future Oncol. 2019;15(26):3033-3045. doi:10. 2217/fon-2019-0029.

10. Wang T, Gao T, Yang J, et al. Preoperative prediction of pelvic lymph nodes metastasis in early-stage cervical cancer using radiomics nomogram developed based on T2-weighted MRI and diffusion-weighted imaging. Eur $J$ Radiol. 2019;114:128-135. doi:10.1016/j.ejrad.2019.01.003

11. Kong TW, Kim J, Son JH, et al. Preoperative nomogram for prediction of microscopic parametrial infiltration in patients with FIGO stage IB cervical cancer treated with radical hysterectomy. Gynecol Oncol. 2016;142(1):109-114. doi:10.1016/j.ygyno.2016.05.010

12. Torre LA, Bray F, Siegel RL, Ferlay J, Lortet-Tieulent J, Jemal A. Global cancer statistics, 2012. CA Cancer J Clin. 2015;65(2):87-108. doi:10.3322/caac. 21262

13. Kaneko N. Factors associated with cervical cancer screening among young unmarried Japanese women: results from an internet-based survey. BMC Women's Health. 2018;18(1):132.

14. Chang C, Chen J, Chang WY, Chiang AJ. Tumor size has a time-varying effect on recurrence in cervical cancer. $J$ Low Genit Tract Dis. 2016;20(4):317-320. doi:10.1097/LGT.000000000000 0238

15. Wang J, Wang T, Yang YY, Chai YL, Shi F, Liu ZI. Patient age, tumor appearance and tumor size are risk factors for early recurrence of cervical cancer. Mol Clin Oncol. 2015;3(2):363-366. doi:10.3892/ mco.2014.465

16. Moore DH, Tian C, Monk BJ, Long HJ, Omura GA, Bloss JD. Prognostic factors for response to cisplatin-based chemotherapy in advanced cervical carcinoma: a Gynecologic Oncology Group Study. Gynecol Oncol. 2010;116(1):44-49. doi:10.1016/j.ygyno.2009. 09.006

17. Zhou J, Zhang WW, Wu SG, et al. The impact of examined lymph node count on survival in squamous cell carcinoma and adenocarcinoma of the uterine cervix. Cancer Manag Res. 2017;9:315-322. doi:10.2147/CMAR.S141335

18. Fleming ND, Frumovitz M, Schmeler KM, et al. Significance of lymph node ratio in defining risk category in node-positive early stage cervical cancer. Gynecol Oncol. 2015;136(1):48-53. doi:10.1016/j.ygyno.2014.11.010.

19. Koh WJ, Greer BE, Abu-Rustum NR, et al. Cervical cancer, version 2.2015. J Natl Compr Canc Netw. 2015;13(4):395-404; quiz 404. doi:10.6004/jnccn.2015.0055.

20. Huang K, Jia M, Li P, et al. Radiotherapy improves the survival of patients with metastatic cervical cancer: a propensity-matched analysis of SEER database. Int J Gynecol Cancer. 2018;28(7):1360-1368. doi:10.1097/IGC.0000000000001313.

21. Jhawar S, Hathout L, Elshaikh MA, Beriwal S, Small W Jr, Mahmoud O. Adjuvant chemoradiation therapy for cervical cancer and effect of timing and duration on treatment outcome. Int $J$ Radiat Oncol Biol Phys. 2017;98(5):1132-1141. doi:10.1016/j.ijrobp.2017. 03.045

22. Frumovitz M, Querleu D, Gil-Moreno A, et al. Lymphadenectomy in locally advanced cervical cancer study (LiLACS): Phase III clinical trial comparing surgical with radiologic staging in patients with stages IB2-IVA cervical cancer. J Minim Invasive Gynecol. 2014;21 (1):3-8. doi:10.1016/j.jmig.2013.07.007

23. Xie Y, Zhao FH, Lu SH, et al. Assessment of quality of life for the patients with cervical cancer at different clinical stages. Chin J Cancer. 2013;32(5):275-282. doi:10.5732/cjc.012.10047 


\section{Publish your work in this journal}

The International Journal of General Medicine is an international, peer-reviewed open-access journal that focuses on general and internal medicine, pathogenesis, epidemiology, diagnosis, monitoring and treatment protocols. The journal is characterized by the rapid reporting of reviews, original research and clinical studies across all disease areas. The manuscript management system is completely online and includes a very quick and fair peer-review system, which is all easy to use. Visit http://www.dovepress.com/ testimonials.php to read real quotes from published authors. 